\title{
LITERACIA EM SAÚDE MENTAL POSITIVA NOS ENFERMEIROS DE CUIDADOS DE SAÚDE PRIMÁRIOS
}

\author{
Amadeu Gonçalves \\ Politécnico de Viseu, Escola Superior de Saúde \\ CINTESIS, Portugal \\ agoncalvessv@hotmail.com \\ Lídia Cabral \\ Politécnico de Viseu, Escola Superior de Saúde \\ CI\&DETS, Portugal \\ alcabral@essv.ipv.pt \\ Carla Cruz \\ Politécnico de Viseu, Escola Superior de Saúde \\ CI\&DETS, Portugal \\ alcabral@essv.ipv.pt \\ Cláudia Chaves \\ Politécnico de Viseu, Escola Superior de Saúde \\ Ci\&DEl, Portugal \\ claudiachaves21@gmail.com \\ Carlos Sequeira \\ Escola Superior de Enfermagem do Porto \\ CINTESIS, Portugal \\ carlossequeira@esenf.pt \\ José Figueiredo Rodrigues \\ Equipa de Tratamento do Centro De Respostas \\ Integradas de Viseu, Portugal \\ zezitodez@hotmail.com
}

Recepción Artículo: 15 mayo 2021 Admisión Evaluación: 15 mayo 2021 Informe Evaluador 1: 17 mayo 2021 Informe Evaluador 2: 21 mayo 2021 Aprobación Publicación: 02 junio 2021

\section{RESUMO}

Introdução: A literacia em saúde mental é fundamental para o reconhecimento de problemas de saúde mental e intervenção precoce. A sua avaliação é importante para identificar deficits de conhecimento sobre a saúde/doença mental e ajudar na definição de programas de intervenção para a promover a literacia em saúde mental, bem como para a avaliação dessas intervenções. Objetivos: Identificar que variáveis sociodemográficas e contextuais à história de doença e comportamentos de saúde interferem na saúde mental positiva dos enfermeiros dos cuidados de saúde primários; verificar se existe relação entre a literacia em saúde mental e a saúde mental positiva dos enfermeiros dos cuidados de saúde primários. Métodos: Estudo transversal, descritivo-correlacio- 


\section{LITERACIA EM SAÚDE MENTAL POSITIVA NOS ENFERMEIROS DE CUIDADOS DE SAÚDE PRIMÁRIOS}

nal e de natureza quantitativa numa amostra de 89 enfermeiros a exercerem funções num agrupamento de centros de saúde da região centro de portugal (ACES-Dão Lafões). 0 instrumento de colheita de dados incluiu um questionário com questões relativas á caracterização sociodemográfica, história de doença mental e comportamentos de saúde. A Escala de Literacia em Saúde Mental (0 Conner et al., 2015) adaptada e validada para a população portuguesa por Loureiro e Carvalho (2018), Questionário de Saúde Mental Positiva traduzido e adaptado para a população portuguesa por Sequeira, Carvalho, Sampaio, Sá, Lluch-Canut e Roldán-Merino (2014) e a Escala 0 que é importante para uma boa saúde mental? (Chaves, Sequeira \& Duarte, 2019). Resultados: Os resultados do estudo mostram um predomínio do género feminino ( $82,0 \%$ ), com uma idade média de $45,60 \pm 8,43$ anos. Pela ordenação dos valores da média os enfermeiros da amostra possuem níveis elevados de literacia em saúde mental $(67,06 \pm 7.73)$. Os valores médios mais elevados foram encontrados nas dimensões: Reconhecimento e auto ajuda apropriada $(\mathrm{M}=71,34 \pm 10,58)$ e Conhecimento sobre apoio profissional disponivel $(\mathrm{M}=68.41 \pm 16.74)$. A análise da relação entre a saúde mental positiva e a literacia em saúde mental revela que os enfermeiros com literacia crítica apresentam melhores níveis de saúde mental positiva. As variáveis Preditores da saúde mental positiva foram: 0 sexo masculino, idade, os anos de experiência profissional, as horas de sono e todas as dimensões da literacia em saúde mental que explicam $24,8 \%$ da variação. Conclusão: Os enfermeiros que participaram no estudo possuem níveis elevados de Literacia em Saúde Mental (Literacia Critica), com associações positivas e significativas com a saúde mental positiva. Esta investigação, mostra algumas das implicações que podem resultar de baixos níveis de literacia em saúde mental, podendo impedir o reconhecimento precoce dos sintomas e adiar a procura de ajuda. Os resultados encontrados trazem contributos importantes para a criação de programas de intervenção em literacia em saúde mental nos profissionais dos cuidados de saúde primários. Investir nesta área, junto dos enfermeiros que prestam cuidados de proximidade (CSP) aumenta a eficácia das suas intervenções de promoção da saúde mental.

Palavras-chave: enfermeiro; cuidados de saúde primários; literacia em saúde mental; saúde mental positiva

\section{ABSTRACT}

Introduction: Mental health literacy is fundamental for the recognition of mental health problems and early intervention. Its assessment is important to identify knowledge deficits about mental health/mental illness and help define intervention programs to promote mental health literacy, as well as to evaluate these interventions. Objectives: To identify which sociodemographic and contextual variables to illness history and health behaviors interfere with the positive mental health of primary health care nurses; to verify whether there is a relationship between mental health literacy and the positive mental health of primary health care nurses. Methods: Cross-sectional, descriptive and correlational study with a quantitative nature in a sample of 89 nurses working in a cluster of health care centers in the central region of Portugal (ACES-Dão Lafões). The data collection instrument included a questionnaire with questions on sociodemographic characterization, history of mental illness and health behaviors. The Mental Health Literacy Scale (O'Conner et al., 2015) adapted and validated for the Portuguese population by Loureiro and Carvalho (2018), the Positive Mental Health Questionnaire translated and adapted for the Portuguese population by Sequeira, Carvalho, Sampaio, Sá, Lluch-Canut, and Roldán-Merino (2014), and the What is important for a good mental health? (Chaves, Sequeira \& Duarte, 2019). Results: The results of the study show a predominance of the female gender $(82.0 \%)$, with a mean age of $45.60 \pm 8.43$ years. By the ordering of the mean values the nurses in the sample have high levels of mental health literacy $(67.06 \pm 7.73)$. The highest mean values were found in the dimensions: Recognition and appropriate self-help ( $M=71.34 \pm 10.58)$ and Knowledge about available professional support $(M=68.41 \pm 16.74)$. Analysis of the relationship between positive mental health and mental health literacy reveals that nurses with critical literacy have better levels of positive mental health. The variables Predictors of positive mental health were: Male gender, age, years of professional experience, hours of sleep and all dimensions of mental health literacy which explained $24.8 \%$ of the variance. Conclusion: The nurses who participated in the study have high levels of Mental Health Literacy (Critical Literacy), with positive and significant associations with positive mental health. This research, shows some of the 
implications that may result from low levels of mental health literacy, which may prevent early recognition of symptoms and delay help-seeking. The findings make important contributions to the creation of mental health literacy intervention programs for primary health care professionals. Investing in this area with primary care nurses increases the effectiveness of their mental health promotion interventions.

Keywords: nurse; primary health care; mental health literacy; positive mental health

\section{INTRODUÇÃO}

Neste artigo abordam-se alguns conceitos chave inerentes à contextualização do estudo, designadamente: saúde mental, literacia em saúde mental e saúde mental positiva e são apresentados os resultados da investigação empírica realizada numa amostra de Enfermeiros dos Cuidados de Saúde Primários. Os Cuidados de Saúde Primários formados por "unidades de saúde muito próximas da população" representam um contexto privilegiado de prestação de cuidados de proximidade, de promoção da saúde e prevenção da doença ao longo do ciclo de vida da pessoa e da sua família.

Bons níveis de literacia em saúde mental são determinantes para o diagnóstico de situações de doença e comportamentos de procura de ajuda. 0 conceito de literacia em saúde mental, decorrente do conceito de literacia em saúde, é definido como a capacidade de obter e manter uma saúde mental positiva, compreender problemas de saúde mental e os seus tratamentos; diminuição do estigma relacionado com os problemas de saúde mental e aprimorar a eficácia na procura de ajuda (England leads the National Health Service England, 2019).

A literacia em saúde mental, aumenta a resiliência e desenvolve nas pessoas habilidades de autogestão, ajudando-as a gerir melhor sua condição de saúde/doença mental a longo prazo. Melhorar a literacia em saúde mental é capacitar as pessoas para tomarem decisões informadas sobre a sua saúde mental e das suas famílias.

0 conceito de saúde mental positiva (SM+) surge como elemento que integra a saúde global da pessoa, cuja terminologia 'positiva' se refere à promoção de ações que reforçam e potenciam a saúde mental na globalidade (Lluch, 2008). A saúde mental positiva pode ser compreendida como um valor em si, ou seja, "sentir-se bem", ou como "uma capacidade para perceber, compreender e interpretar o meio, para se adaptar e alterá-Io, se necessário, para pensar e comunicar com os outros (Sequeira, Carvalho, Sampaio, Lluch-Canut \& Roldán-Merino, 2014, p. 46).

A promoção da saúde mental positiva maximiza a qualidade de vida e o bem-estar psicológico quer das pessoas com problemas de saúde mental e dos seus cuidadores, quer da população em geral, possibilitando o seu empowerment para que se sintam com capacidade de aumentar o controlo sobre a própria saúde, lidarem com as adversidades e realizarem escolhas mais informadas, em relação ao seu próprio comportamento, adotando estilos de vida saudáveis.

Mais recentemente, o conceito de literacia em saúde mental foi definido como a forma de compreender como obter e manter a saúde mental positiva; compreender os transtornos mentais e os seus tratamentos; a diminuição do estigma relacionado com os transtornos mentais; melhorar a eficácia de procura de ajuda, saber quando e onde procurar ajuda e desenvolver competências destinadas a melhorar os recursos de cuidados de saúde mental e de autogestão (Kutcher, Wei \& Connie, 2016).

\section{CONSIDERAÇÕES METODOLÓGICAS}

\section{Tipo de Estudo}

A literacia em saúde mental positiva, é uma competência fundamental nos Enfermeiros de Cuidados de Saúde Primários, ela promove o autoconhecimento e autocontrolo, tão importantes numa relação teraputica e nas intervenções de promoção da saude mental que desenvolvem junto dos individuos, familias e comunidade. Neste sentido, desenvolveu-se um estudo de natureza quantitativa, transversal, descritivo e correlacional, numa amostra não probabilística por conveniência constituída por 89 Enfermeiros a exercerem funções num agrupamento de centros de saúde da região centro de Portugal (ACEs Dão Lafões). 


\section{LITERACIA EM SAÚDE MENTAL POSITIVA NOS ENFERMEIROS DE CUIDADOS DE SAÚDE PRIMÁRIOS}

\section{Instrumento de colheita de dados}

Na colheita de dados, foi utilizado um questionário de autopreenchimento, com questões relativas á caracterização sociodemográfica e profissional dos enfermeiros; história de doença mental e comportamentos de saúde. A Escala de Literacia em Saúde Mental (0 Conner et al., 2015) adaptada e validada para a população portuguesa por Loureiro e Carvalho (2018), composta por 35 questões distribuídas pelas seguintes dimensões: Habilidade para reconhecer transtornos mentais (itens 1 a 8); Conhecimento sobre causas e fatores de risco (itens 9 e 10); Conhecimento sobre apoio profissional disponível (itens 11 a 13); Conhecimento sobre estratégias de autoajuda (itens 14-15); Conhecimento sobre as formas de procurar informação sobre a saúde (itens 16 a 19); Atitudes que promovem reconhecimento e auto-ajuda apropriada (itens 20 a 28, itens com cotação inversa) e Atitudes que promovem reconhecimento e auto-ajuda apropriada (itens 29 a 35). Apresenta propriedades psicométricas consideráveis e é facilmente administrado e mensurável. Segundo os autores da escala, quanto maior a pontuação global obtida melhor o nível de literacia em saúde mental do individuo avaliado. Consideraram-se como pontos de corte indicativos do nível de literacia os seguintes valores: 35-76 (literacia básica funcional); 77-118 (literacia comunicativa/interativa); e 119- 160 (literacia crítica). (Loureiro \& Carvalho, 2018, p. 55).

Foi aplicado o Questionário de Saúde Mental Positiva traduzido e adaptado à população portuguesa por Sequeira, Carvalho, Sampaio, Sá, Lluch-Canut e Roldán-Merino (2014), que obtiveram resultados consistentes para a fidelidade, validade de conteúdo e validade de critério quando utilizado em amostras de população adulta portuguesa. Esta escala é pontuada de 1 a 4, sendo o limite superior do QSM+ de 39 e 0 inferior de 156. Deve-se considerar que a pontuação obtida no somatório do QSM+ é inversamente proporcional à SM+. Assim, quanto menor a pontuação global no QSM+ melhores serão os níveis de SMP. 0 estudo psicométrico da escala revelou boa consistência interna $(=0,905)$. A Escala $\mathbf{O}$ que é importante para uma boa saúde mental? (Chaves, Sequeira \& Duarte, 2019), constituída por 10 declarações sobre as coisas que podem ser importantes para uma boa saúde mental, avaliadas numa escala de tipo Likert de 1 a 5 , em que 1 corresponde a "Discordo totalmente" e 5 a "Concordo totalmente". Contém ainda a opção "Não aplicável", que corresponde a 0 (zero).

\section{Estudo psicométrico dos Instrumentos utilizados}

Foram estudadas as estatísticas (médias e desvios padrão), assim como as correlações entre cada item e 0 valor global da Escala 0 que é importante para uma boa saúde mental?, permitindo observar como cada item se relaciona com o valor global.

Para o estudo da homogeneidade dos itens, foi realizado o cálculo das estatísticas descritivas, assim como as correlações em cada item e 0 valor global de cada escala. Os valores de Alfa de Cronbach dos itens oscilam entre 0,827 nos itens 6 e 7 e 0,868 no item 4, indicando boa consistência interna. Os coeficientes de correlação do item total corrigido apontam para uma correlação máxima situada no item 7 "Dominar os seus pensamentos negativos" ( $r=0,758)$. Os valores da média e respetivo desvio padrão, nos diversos itens, permitem afirmar que estes se encontram bem centrados, uma vez que todos se situam acima da média esperada, sendo o menor no item $4(M=4,20)$ e o maior no item $2(M=4,73)$. Encontrou-se um alfa global de $0,855,0$ que significa que a escala tem boa consistência interna.

\section{Procedimentos éticos e formais}

Pediu-se autorização ao ACeS Dão Lafões para aplicação dos questionários, tendo sido favorável. Foi entregue a cada enfermeiro, juntamente com o questionário, um pedido de consentimento informado. Os questionários foram entregues em envelopes a cada instituição (21) e o protocolo de recolha dos questionários seguiu o mesmo procedimento.

\section{Tratamento estatístico dos dados}

Após a colheita de dados utilizou-se o programa SPSS versão 25.0 para a criação da base de dados e tratamento estatístico. A estatística descritiva possibilitou determinar as frequências absolutas e percentuais, algumas 
medidas de tendência central, nomeadamente as médias e as medidas de dispersão, como a amplitude de variação, o coeficiente de variação e 0 desvio padrão.

$\mathrm{Na}$ estatística inferencial, recorreu-se a testes paramétricos e não paramétricos, nomeadamente: Testes t de Student ou teste de U Mann Whitney; Análise de Teste ANOVA ou Kruskall Wallis e Teste de qui quadrado ( $X^{2}$ )

\section{APRESENTAÇÃO DOS RESULTADOS}

Caracterização sociodemográfica e profissional da amostra

Relativamente ao perfil sociodemográfico e profissional dos Enfermeiros que participaram no estudo (89 enfermeiros), os resultados mostram que a maioria é do género feminino (82,0\%), com uma idade mínima de 28 anos e uma máxima de 64 anos, ao que corresponde uma idade média de 45,60 anos ( $\pm 8,43$ anos), sobressaindo os que possuem idade compreendida entre os $41-49$ anos (34,8\%), com companheiro(a) (77,5\%), licenciados $(67,4 \%)$, que não estão deslocados da sua residência $(86,5 \%)$, com mais tempos de exercício profissional ( $\geq 26$ anos) $(34,8 \%)$, com horário semanal $\leq 35$ horas $(83,1 \%)$. 
Tabela 1 - Caracterização sociodemográfica e profissional em função do género

\begin{tabular}{|c|c|c|c|c|c|c|c|c|c|c|}
\hline \multirow{2}{*}{$\begin{array}{l}\text { Género } \\
\text { Variáveis }\end{array}$} & \multicolumn{2}{|c|}{ Masculino } & \multicolumn{2}{|c|}{ Feminino } & \multicolumn{2}{|c|}{ Total } & \multicolumn{2}{|c|}{ Residuais } & \multirow[t]{2}{*}{$\mathrm{X}^{2}$} & \multirow[t]{2}{*}{$\mathbf{p}$} \\
\hline & $\begin{array}{l}\mathrm{n}^{0} \\
(\mathbf{1 6})\end{array}$ & $\begin{array}{c}\% \\
(18.0\end{array}$ & $\begin{array}{c}\mathbf{n}^{\mathbf{0}} \\
(73 \\
)\end{array}$ & $\begin{array}{c}\% \\
(82.0\end{array}$ & $\begin{array}{l}\mathrm{n}^{0} \\
(89\end{array}$ & $\begin{array}{c}\% \\
(100.0\end{array}$ & 1 & 2 & & \\
\hline \multicolumn{11}{|l|}{ Idade } \\
\hline$\leq 40$ anos & 6 & 37.5 & 23 & 31.5 & 29 & 32.6 & .5 & -.5 & 0.227 & 0.893 \\
\hline $41-49$ anos & 5 & 31.3 & 26 & 35.6 & 31 & 34.8 & -.3 & .3 & & \\
\hline$\geq 50$ anos & 5 & 31.3 & 24 & 32.9 & 29 & 32.6 & -.1 & .1 & & \\
\hline \multicolumn{11}{|l|}{ Estado civil } \\
\hline $\begin{array}{l}\text { Sem } \\
\text { companheiro(a) }\end{array}$ & 3 & 18.8 & 17 & 23.3 & 20 & 22.5 & -.4 & .4 & \multicolumn{2}{|c|}{ Não aplicável } \\
\hline $\begin{array}{l}\text { Com } \\
\text { companheiro(a) }\end{array}$ & 13 & 81.3 & 56 & 76.7 & 69 & 77.5 & .4 & -.4 & & \\
\hline $\begin{array}{l}\text { Formação } \\
\text { académica }\end{array}$ & & & & & & & & & & \\
\hline Licenciatura & 11 & 68.8 & 49 & 67.1 & 60 & 67.4 & .1 & -.1 & & \\
\hline Mestrado & 5 & 31.3 & 24 & 32.9 & 29 & 32.6 & -.1 & .1 & 0.016 & 0.900 \\
\hline $\begin{array}{l}\text { Deslocado da } \\
\text { residência }\end{array}$ & & & & & & & & & & \\
\hline $\operatorname{Sim}$ & 2 & 12.5 & 10 & 13.7 & 12 & 13.5 & -.1 & .1 & Não & icável \\
\hline Não & 14 & 87.5 & 63 & 86.3 & 77 & 86.5 & .1 & -.1 & & \\
\hline $\begin{array}{ll}\text { Tempo } & \text { de } \\
\text { exercício } & \text { na } \\
\text { profissão } & \end{array}$ & & & & & & & & & & \\
\hline$\leq 16$ anos & 7 & 43.8 & 23 & 31.5 & 30 & 33.7 & .9 & -.9 & & \\
\hline$\overline{17}-25$ anos & 5 & 31.3 & 23 & 31.5 & 28 & 31.5 & .0 & .0 & 1.125 & 0.570 \\
\hline$\geq 26$ anos & 4 & 25.0 & 27 & 37.0 & 31 & 34.8 & -.9 & .9 & & \\
\hline $\begin{array}{l}\text { Abstinência } \\
\text { laboral no } \\
\text { último ano }\end{array}$ & & & & & & & & & & \\
\hline Sim & 7 & 43.8 & 21 & 28.8 & 28 & 31.5 & 1.2 & -1.2 & 1.366 & 0.242 \\
\hline Não & 9 & 56.3 & 52 & 71.2 & 61 & 68.5 & -1.2 & 1.2 & & \\
\hline $\begin{array}{lr}\text { Número } & \text { de } \\
\text { horas } & \text { por } \\
\text { semana } & \end{array}$ & & & & & & & & & & \\
\hline$\leq 35$ horas & 14 & 87.5 & 60 & 82.2 & 74 & 83.1 & .5 & -.5 & Não c & icável \\
\hline$\geq 36$ horas & 2 & 12.5 & 13 & 17.8 & 15 & 16.9 & -.5 & .5 & & \\
\hline Total & 16 & 100.0 & 73 & 100.0 & 89 & 100.0 & & & & \\
\hline
\end{tabular}

Em relação ao tempo de exercício na profissão, os resultados revelam, para a globalidade da amostra, um mínimo de 2 anos e um máximo de 44 anos, ao que corresponde uma média 22,19 anos ( $\pm 8,56$ anos). Para os Enfermeiros, 0 tempo mínimo de exercício na categoria é 12 anos e um máximo de 44 anos e, para as Enfermeiras, o mesmo oscila entre 2 e os 41 anos. As Enfermeiras, em média ( $M=22,40$ anos $\pm 8,51$ anos), têm ligeiramente mais tempo de exercício na profissão do que os Enfermeiros ( $M=21,25$ anos $\pm 9,05$ anos). Os coeficientes de variação indiciam dispersão entre 0 baixo e 0 elevado face às médias encontradas. 
Tabela 3 - Estatísticas relativas aos anos de exercício na profissão o género

\begin{tabular}{lcccccccc}
\hline Género & $\mathrm{N}$ & Min & Max & M & DP & CV (\%) & Sk/erro & K/erro \\
\hline Masculino & 16 & 12 & 44 & 21.25 & 9.05 & 42.58 & 2.35 & 1.15 \\
Feminino & 73 & 2 & 41 & 22.40 & 8.51 & 15.36 & 0.25 & -1.16 \\
Total & $\mathbf{8 9}$ & $\mathbf{2}$ & $\mathbf{4 4}$ & $\mathbf{2 2 . 1 9}$ & $\mathbf{8 . 5 6}$ & $\mathbf{3 8 . 5 7}$ & $\mathbf{1 . 1 3}$ & $\mathbf{- 0 . 9 6}$ \\
\hline
\end{tabular}

\section{Comportamentos de saúde}

Os resultados encontrados, relativos aos comportamentos de saúde mostram que $55,1 \%$ dos enfermeiros estão satisfeitos com o seu sono; $52,8 \%$ consideraram que dormem as horas suficientes para as suas necessidades. A grande maioria dos enfermeiros (88,8\%) não toma medicação para dormir e não tomam medicação de forma regular para problemas de saúde mental $(92,1 \%)$.

Tabela 2 - Variáveis de comportamentos de saúde em função do género

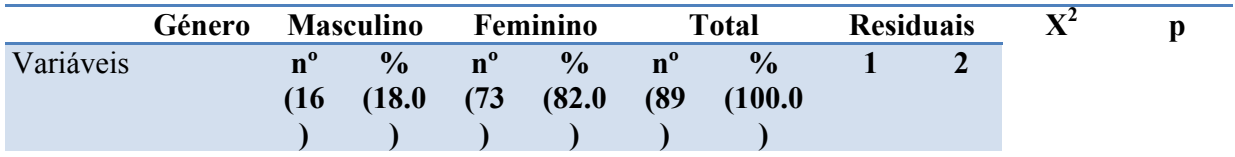

Estar satisfeito

com o seu sono

\begin{tabular}{|c|c|c|c|c|c|c|c|c|c|c|}
\hline Sim & 9 & 56,3 & 40 & 54,6 & 49 & 55.1 & .1 & -.1 & \multirow[t]{2}{*}{0.011} & \multirow[t]{2}{*}{0.916} \\
\hline Não & 7 & 43,8 & 33 & 45,2 & 40 & 44.9 & -.1 & .1 & & \\
\hline $\begin{array}{l}\text { Considerar que } \\
\text { dorme as horas } \\
\text { suficientes para as } \\
\text { suas necessidades }\end{array}$ & & & & & & & & & & \\
\hline Sim & 8 & 50,0 & 39 & 53,4 & 47 & 52.8 & -.2 & .2 & 0.062 & 0.084 \\
\hline Não & 8 & 50,0 & 56 & 46,6 & 42 & 47.2 & .2 & -.2 & & \\
\hline
\end{tabular}

Toma de

medicação para

dormir

\begin{tabular}{lcccccccc} 
Sim & 3 & 18,8 & 7 & 9,6 & 10 & 11.2 & .1 & -.1 \\
Não & 13 & 81,3 & 66 & 90,4 & 79 & 88.8 & -.1 & .1 \\
\hline
\end{tabular}

Toma de

medicação de

forma regular para

algum problema de $\quad$ Não aplicável

saúde mental

$\begin{array}{lcccccccc}\text { Sim } & 0 & 0,0 & 7 & 9,6 & 7 & 7.9 & -1.3 & 1.3 \\ \text { Não } & 16 & 100,0 & 66 & 90,4 & 82 & 92.1 & 1.3 & 1.3\end{array}$

Prática de algum

Não aplicável

desporto ou

exercício físico

regular

$\begin{array}{lcccccccc}\text { Sim } & 11 & 66,8 & 41 & 56,2 & 52 & 58.4 & .9 & -.9 \\ \text { Não } & 5 & 31,3 & 32 & 43,8 & 37 & 41.6 & -.9 & .9\end{array}$

Considerar a sua

Não aplicável

alimentação

saudável 


\section{LITERACIA EM SAÚDE MENTAL POSITIVA NOS ENFERMEIROS DE CUIDADOS DE SAÚDE PRIMÁRIOS}

\begin{tabular}{|c|c|c|c|c|c|c|c|c|c|}
\hline Sim & 14 & 87,5 & 64 & 87,7 & 78 & 87.6 & -.2 & .2 & \\
\hline Não & 2 & 12,5 & 9 & 12,3 & 11 & 12.4 & .2 & -.2 & \\
\hline $\begin{array}{l}\text { Participar em } \\
\text { alguma atividade } \\
\text { recreativa }\end{array}$ & & & & & & & & & \\
\hline Sim & 6 & 37,5 & 8 & 11 & 14 & 15.7 & 2.6 & -2.6 & Não aplicável \\
\hline Não & 10 & 62,5 & 65 & 89.0 & 75 & 84.3 & -2.6 & 2.6 & \multirow{4}{*}{ Não aplicável } \\
\hline $\begin{array}{l}\text { Ter alguma relação } \\
\text { afetiva } \\
\text { significativa }\end{array}$ & & & & & & & & & \\
\hline Sim & 16 & 100.0 & 67 & 91.8 & 83 & 93.3 & 1.2 & -1.2 & \\
\hline Não & 0 & 0.0 & 6 & 8.2 & 6 & 6.7 & -1.2 & 1.2 & \\
\hline $\begin{array}{l}\text { Estar satisfeito } \\
\text { com a sua vida } \\
\text { afetiva }\end{array}$ & & & & & & & & & \multirow[t]{3}{*}{ Não aplicável } \\
\hline Sim & 15 & 93.8 & 69 & 94.5 & 84 & 94.4 & -.1 & .1 & \\
\hline Não & 1 & 6.3 & 4 & 5.5 & 5 & 5.6 & .1 & -.1 & \\
\hline
\end{tabular}

\section{Saúde mental Positiva}

Os valores obtidos na escala de saúde mental positiva são baixos $(65,89 \pm 14,01)$. 0 que nos permite afirmar que os níveis de saúde mental positiva nos enfermeiros são bons na medida em que a pontuação obtida no somatório do QSM+é inversamente proporcional à SM+. Assim, quanto menor a pontuação global no QSM+ melhores serão os níveis de SMP.

Os valores médios mais elevados correspondem à resolução de problemas e realização pessoal $(M=15,61 \pm 4,74)$, satisfação pessoal $(12,44 \pm 3,57)$ e habilidade de relação interpessoal ( $M=12,34 \pm 3,17)$ e a menor regista-se na atitude pró-social ( $M=7,40 \pm 2,14)$.

Tabela 3 - Estatísticas relativas à saúde mental positiva dos Participantes

\begin{tabular}{|c|c|c|c|c|c|}
\hline Dimensões /fatores & Min & Max & $\mathbf{M}$ & D.P. & $\begin{array}{l}\text { CV } \\
(\%)\end{array}$ \\
\hline Satisfação pessoal & 8.00 & 22.00 & 12.44 & 3.57 & 28.69 \\
\hline Atitude pró-social & 5.00 & 13.00 & 7.40 & 2.14 & 28.91 \\
\hline Autocontrolo & 5.00 & 15.00 & 9.61 & 2.40 & 24.97 \\
\hline Autonomia & 5.00 & 13.00 & 8.46 & 2.04 & 24.11 \\
\hline $\begin{array}{l}\text { Resolução de problemas e realização } \\
\text { pessoal }\end{array}$ & 9.00 & 27.00 & 15.61 & 4.74 & 30.36 \\
\hline Habilidade de relação interpessoal & 7.00 & 21.00 & 12.34 & 3.17 & 25.68 \\
\hline $\begin{array}{l}\text { Saúde mental positiva (fator } \\
\text { global) }\end{array}$ & 44.00 & 122.0 & 65.89 & 14.01 & 21.26 \\
\hline
\end{tabular}

\section{Literacia em saúde mental}

Pela ordenação dos valores da média os enfermeiros da amostra possuem níveis elevados de literacia em saúde mental ( 67,06 \pm 7.73$)$. Os valores médios mais elevados correspondem ás Atitudes que promovem reconhecimento e auto ajuda apropriada $(\mathrm{M}=71.34 \pm 10,58)$ e Conhecimento sobre apoio profissional disponivel ( $\mathrm{M}=68.41 \pm 16.74)$ 
Tabela 4 - Estatísticas relativas à Literacia em saúde mental positiva dos Participantes

\begin{tabular}{lccccc}
\hline Dimensões /fatores & Min & Max & M & D.P. \\
\hline $\begin{array}{l}\text { Habilidade para reconhecer transtornos } \\
\text { mentais }\end{array}$ & 25.00 & 83.30 & 60.15 & 13.79 \\
$\begin{array}{l}\text { Conhecimento sobre causas e fatores de } \\
\text { risco }\end{array}$ & 16.67 & 100 & 59.55 & 15.26 \\
$\begin{array}{l}\text { Conhecimento sobre apoio profissional } \\
\text { disponível }\end{array}$ & 33.33 & 100 & $\mathbf{6 8 . 4 1}$ & 16.74 \\
$\begin{array}{l}\text { Conhecimento sobre estratégias de } \\
\text { autoajuda }\end{array}$ & 16.67 & 100 & 57.49 & 14.65 \\
$\begin{array}{l}\text { Conhecimento sobre as formas de procurar } \\
\text { informação sobre a saúde }\end{array}$ & 25.00 & 100 & 65.94 & 16.42 \\
$\begin{array}{l}\text { Atitudes que promovem reconhecimento e } \\
\text { auto ajuda apropriada }\end{array}$ & 42.19 & 100 & $\mathbf{7 1 . 3 4}$ & 10.58 \\
$\begin{array}{l}\text { Literacia em Saúde mental } \\
\text { global) }\end{array}$ & (fator & 51.20 & 91.20 & $\mathbf{6 7 . 0 6}$ & 7.73 \\
\hline
\end{tabular}

\section{Relação entre a saúde mental positiva e a literacia em saúde mental}

Os Participantes possuem níveis elevados de Literacia em Saúde Mental (Literacia Critica). A análise da relação entre a saúde mental positiva e a literacia em saúde mental revela que os enfermeiros com literacia crítica pontuaram menos, à exceção da autonomia, sugerindo que são estes os que apresentam melhores níveis de saúde mental positiva, com diferenças estatisticamente significativas na atitude pró-social $(\mathrm{p}=0,028)$

Teste Tabela 5 - Teste U de Mann-Whitney entre a saúde mental positiva e a literacia em saúde

\begin{tabular}{|c|c|c|c|c|}
\hline Literacia em saúde & $\begin{array}{l}\text { Literacia } \\
\text { interativa }\end{array}$ & $\begin{array}{l}\text { Literacia } \\
\text { crítica }\end{array}$ & \multirow[t]{2}{*}{ UMW } & \multirow[t]{2}{*}{$\mathbf{p}$} \\
\hline Saúde mental positiva & $\begin{array}{l}\text { Ordenação } \\
\text { Média }\end{array}$ & $\begin{array}{l}\text { Ordenação } \\
\text { Média }\end{array}$ & & \\
\hline Satisfação pessoal & 45.90 & 44.19 & .100 & .752 \\
\hline Atitude pró-social & 51.27 & 39.39 & 4.844 & .028 \\
\hline Autocontrolo & 47.96 & 42.35 & 1.070 & .301 \\
\hline Autonomia & 44.19 & 45.72 & .080 & .778 \\
\hline $\begin{array}{l}\text { Resolução de problemas } \mathrm{e} \\
\text { realização pessoal }\end{array}$ & 48.30 & 42.05 & 1.303 & .254 \\
\hline Habilidade de relação interpessoal & 49.06 & 41.37 & 1.984 & .159 \\
\hline $\begin{array}{l}\text { Saúde mental positiva (fator } \\
\text { global) }\end{array}$ & 48.42 & 41.95 & 1.392 & .238 \\
\hline
\end{tabular}

\section{DISCUSSÃO DOS RESULTADOS}

A maioria dos enfermeiros da amostra é do género feminino (82,0\%), com uma idade mínima de 28 anos e uma máxima de 64 anos, ao que corresponde uma idade média de 45,60 anos ( $\pm 8,43$ anos), sobressaindo os que possuem idade compreendida entre os $41-49$ anos (34,8\%). No estudo realizado por Cabral e Florentim (2015) também houve um predomínio de enfermeiros do género feminino e com idades acima dos 40 anos a exercerem em cuidados de saúde primários. 


\section{LITERACIA EM SAÚDE MENTAL POSITIVA NOS ENFERMEIROS DE CUIDADOS DE SAÚDE PRIMÁRIOS}

A análise da relação entre a saúde mental positiva e a literacia em saúde mental, que se constituiu como questão central da investigação, revela que os enfermeiros com literacia crítica pontuaram menos, à exceção da autonomia, sugerindo que são estes os que apresentam melhores níveis de saúde mental positiva, com diferença estatisticamente significativa na atitude pró-social $(p=0,028)$. Estes resultados podem ser justificados com o facto de a literacia crítica em saúde se referir às competências cognitivas mais avançadas que, juntamente com as capacidades sociais, podem ser usadas para analisar de forma crítica a informação e usá-la para exercer maior controlo sobre as situações da vida (Pedro, Amaral \& Escoval, 2016).

No que se refere à primeira questão de investigação que previa saber quais as variáveis sociodemográficas que interferem na saúde mental positiva dos enfermeiros dos cuidados de saúde primários, constatou-se que a idade, os anos de experiência profissional e o número de horas de trabalho por semana foram as únicas com relevância estatisticamente significativa. Constatou-se que os enfermeiros com mais idade apresentam valores de ordenação média mais baixos em quase todos os fatores da saúde mental positiva, excetuando a satisfação pessoal, onde pontuaram menos os enfermeiros na faixa etária dos 41-49 anos, com diferenças estaticamente significativas nas habilidades de relação interpessoal $(p=0,019)$ e na saúde mental positiva global $(p=0,012)$, onde se destacam os enfermeiros mais novos. Assim, pode dizer-se que, na globalidade, são os enfermeiros com mais idade os que têm melhor saúde mental positiva. No estudo de Cabral e Florentim (2015), apesar de apenas terem estudado a saúde mental, também não encontraram associações com significância estatística entre a saúde mental dos enfermeiros e as variáveis sociodemográficas e profissionais estudadas.

Em relação à história de saúde mental, a grande maioria (89,9\%) não refere problemas de saúde mental; 95,5\% diz não ter recorrido (nos últimos 3 meses), a qualquer serviço de saúde devido a problemas de saúde mental; 82,0\% negam a necessidade de acompanhamento psicológico ou psiquiátrico e 73,0\% não tiveram ou têm familiares com doença mental. Num estudo realizado em 2019 por (Carvalho, Querido, Tomás \& Cordeiro) numa amostra de 83 enfermeiros, verificaram que a saúde mental dos enfermeiros era razoável, com valores acima do ponto de corte mas apontava para a possível existência de sintomas moderados de sofrimento psicológico, com registo de sintomas de depressão a rondar os 20,5\% e que as mulheres apresentam níveis mais baixos de saúde mental do que os homens. Os mesmos autores referiam ainda que dois terços dos enfermeiros tinham uma percepção negativa da sua saúde mental.

Os enfermeiros com mais saúde mental positiva são os que têm entre 17-25 anos de tempo de exercício profissional, com relevância estatisticamente significativa na satisfação pessoal ( $p=0,018)$. Também revelam melhores níveis de saúde mental positiva os enfermeiros que trabalham $\geq 36$ horas por semana, com diferença estatisticamente significativa na resolução de problemas e realização pessoal $(p=0,043)$. Assim, poder-se-á dizer que os enfermeiros com mais experiência profissional e, apesar de trabalharem mais horas semanais, poderão ter mais saúde mental positiva decorrente da sua resiliência e provavelmente porque já têm estratégias de coping mais adaptadas para enfrentarem o seu quotidiano profissional.

Esta investigação revela também que as variáveis contextuais à história de doença mental que interferem na saúde mental positiva dos enfermeiros são a ausência de problemas de saúde mental, com diferenças estatisticamente significativas em quase todos os fatores e saúde mental positiva fator global $(p<0,05)$, excetuando a atitude pro-social $(p=0,141)$ e autocontrolo $(p=165)$. Os enfermeiros que nunca tiveram necessidade de acompanhamento psicológico ou psiquiátrico também revelam níveis de saúde mental positiva mais elevados, com diferença estatisticamente significativa na satisfação pessoal $(p=0,008)$. Num estudo realizado em 2017 pelo "Centro de Investigação Interdisciplinar em Saúde" (CIIS) da Escola de Enfermagem da Universidade Católica de Lisboa sobre a saúde mental dos enfermeiros portugueses, numa amostra de 1264 participantes, revela que quase dois terços $(60,6 \%)$ relataram uma perceção negativa da sua saúde mental. Estes dados assumem-se como importantes, na medida em que os resultados do nosso estudo vão em sentido contrário, mostrando que a maioria dos enfermeiros não evidenciou problemas de saúde mental, o que poderá justificar os resultados encontrados em relação à saúde mental positiva. 
Quando procuramos saber quais as variáveis de comportamentos de saúde (último mês) que interferem na saúde mental positiva, verificamos que os enfermeiros que estão satisfeitos com o seu sono revelam melhores níveis de saúde mental positiva, com diferença estatisticamente significativa no autocontrolo ( $p=0,019)$, bem como os que não tomam qualquer medicação para dormir, com diferença estatisticamente significativa na satisfação pessoal ( $p=0,011)$ e aqueles que não tomam medicação de forma regular para problemas de saúde mental, com diferença estatisticamente significativa na satisfação pessoal ( $p=0,005)$. Estes resultados consubstanciam os anteriormente referidos, ou seja, as patologias mentais afetam e alteram todas as dimensões que integram o ser humano, mental, física e socialmente.

Como refere Sequeira (2015), a saúde mental positiva traduz-se num estado de funcionamento ótimo do ser humano, o que enfatiza a importância da promoção das qualidades da pessoa na otimização do seu potencial. Deste modo, quando os enfermeiros possuem um estado de bem-estar, que thes permite reconhecer as suas capacidades, estão mais aptos para enfrentar o stresse do seu quotidiano, trabalhar de forma produtiva e contribuir para uma melhor prestação de cuidados aos utentes e comunidade.

0 estudo mostra também que são variáveis preditoras da saúde mental positiva o sexo masculino, idade, os anos de experiência profissional, as horas de sono por dia, o total do que é importante para uma boa saúde mental e todas as dimensões da literacia em saúde mental, nomeadamente: habilidade para reconhecer transtornos mentais, conhecimento sobre causas e fatores de risco, conhecimento sobre apoio profissional disponível, conhecimento sobre estratégias de auto-ajuda, conhecimento sobre as formas de procurar informação sobre a saúde e atitudes que promovem reconhecimento e auto-ajuda apropriada.

Por outro lado, os enfermeiros do sexo masculino, mais novos, com menos horas de sono por dia, com menos conhecimento sobre causas e fatores de risco, apoio profissional disponível, estratégias de auto-ajuda, formas de procurar informação sobre a saúde e menores atitudes que promovem reconhecimento e auto-ajuda apropriada revelam mais saúde mental positiva. Estes resultados podem encontrar justificação no fato de os enfermeiros com níveis mais elevados de saúde mental positiva, possam aproveitar melhor todas as atividades em que desejam participar.

\section{CONCLUSÕES}

Os Cuidados de Saúde Primários representam o primeiro nível de contacto com o cidadão e assumem-se como centrais do Sistema de Saúde, numa perspetiva integrada e de articulação com outros serviços para a continuidade de cuidados. São "o pilar central do sistema nacional de saúde, assumindo importantes funções de promoção da saúde, prevenção e prestação de cuidados na doença, continuidade de cuidados e articulação com outros serviços de saúde" (Ministério da Saúde, 2016, p. 14). Para se cumprirem estes desígnios, é importante que os enfermeiros dos Cuidados de Saúde Primários tenham saúde mental positiva, traduzindo-se em mais capacidade para enfrentar as situações stressantes inerentes à sua vida profissional, equilíbrio emocional/controlo emocional, maior tolerância à frustração, ansiedade e stresse, capacidade para entender os sentimentos dos outros, habilidade para dar apoio emocional e habilidade para estabelecer e manter relações interpessoais.

Os resultados do estudo revelam que os enfermeiros da amostra possuem níveis elevados de literacia em saúde mental $(67,06 \pm 7.73)$. A análise da relação entre a saúde mental positiva e a literacia em saúde mental revelam que os enfermeiros com literacia crítica apresentam melhores níveis de saúde mental positiva.

Após uma reflexão sobre os resultados obtidos neste estudo, consideramos que os dados alcançados podem funcionar como um ponto de partida para a concepção, implementação e avaliação de medidas que potenciem melhores índices de saúde mental positiva em enfermeiros de cuidados de saúde primários, promovendo-se a sua saúde global. Estas intervenções podem aumentar a saúde mental positiva dos enfermeiros e por conseguinte, ajuda-Ios a alcançar o seu potencial. A saúde mental positiva não evita apenas problemas de saúde mental, mas também leva a um maior prazer e satisfação com a vida, o que se traduzirá em cuidados de saúde de qualidade. 


\section{LITERACIA EM SAÚDE MENTAL POSITIVA NOS ENFERMEIROS DE CUIDADOS DE SAÚDE PRIMÁRIOS}

No que se refere às limitações encontradas, salienta-se a reduzida dimensão da amostra, que não permite fazer generalização dos resultados encontrados para outras amostras com as mesmas características e a escassez de estudos sobre a literacia em saúde mental positiva dos enfermeiros dos cuidados de saúde primários. Não obstante, fica a certeza de que bons níveis de literacia em saúde mental positiva nos enfermeiros dos cuidados de saúde primários são fundamentais para o aumento da eficácia dos programas de saúde que desenvolvem junto das pessoas famílias e comunidades.

\section{REFERÊNCIAS BIBLIOGRÁFICAS}

Ad Hoc Committee on Health Literacy for the Council on Scientific Affairs of the American Medical Association (1999, 11 de Fevereiro). Health literacy: Report of the council on scientific affairs. Journal of the American Medical Association. doi:10.1001/jama.281.6.552.

Barry, M.M. (2009). Addressing the determinants of positive mental health: Concepts, evidence and practice. International Journal of Mental Health Promotion, 11, 4-17. D0l: https://doi.org/10.1080/ 14623730.2009.9 721788.

Bjørnsen HN, Ringdal R, Espnes GA, Eilertsen MB, Moksnes UK. Exploring MEST: a new universal teaching strategy for school health services to promote positive mental health literacy and mental wellbeing among Norwegian adolescents. BMC Health Serv Res. 2018;18(1):1001. Published 2018 Dec 29. doi:10.1186/s12913-018-3829-8

Bjørnsen, H. N., Ringdal, R., Espnes, G. A., \& Moksnes, U. K. (2017). Positive mental health literacy: Development and validation of a measure among Norwegian adolescents. BMC Public Health, 17, 717. http://dx.doi.org/10.1186/s12889-017- 4733-6.

Cabral, L,.., \& Florentim, R. (2015). Saúde Mental dos Enfermeiros nos Cuidados de Saúde Primários. Millenium, 49 (jun/dez), 195-216. Acedido em https://www.ipv.pt/millenium/Millenium49/11.pdf

Carvalho, D. de., Querido, A., Tomás, C., Gomes, J., \& Cordeiro, M. (2019). A saúde mental dos enfermeiros: um estudo preliminar. Revista Portuguesa de Enfermagem de Saúde Mental; (21), 47-52. Acedido em http://dx.doi.org/10.19131/rpesm.0237

Centro de Investigação Interdisciplinar em Saúde" (CIIS) da Escola de Enfermagem de Lisboa da Universidade Católica Portuguesa (2018). Apresentação do estudo "A Saúde Mental dos Enfermeiros Portugueses". Acedido em https://ics.lisboa.ucp.pt/news/apresentacao-dos-estudo-saude-mental-dos-enfermeiros-portugueses-7126

Direção-Geral da Saúde. (2015). Plano nacional de saúde: Revisão e extensão a 2020. Acedido em: https://pns.dgs.pt/pns-revisao-e-extensao-a-2020

England leads the National Health Service England. (2019). Enabling people to make informed health decisions: Health Literacy. Acedido em https://www.england.nhs.uk/ourwork/patient-participation/health-decisions/

Jorm, A.F. (2015). Why we need the concept of "mental health literacy". Health Commun.; 30, 1166-8.

Kaur, S., Thapar, K., Saini, P. et al. (2016). Myths \& Misconceptions of Mental IIIness and Health Seeking Behaviour of Adults. International Journal of Community Health and Medical Research, Vol.2, 3, 3-9. D0I: 10.21276/ijchmr.2016.2.3.02

Lakdawala, B.M., \& Vankar, G.K. (2016). A study on community attitudes towards the mentally ill among youth in Gujarat. Indian J Mental Health; 3(4), 473-85.

Lluch, M. T. (2008). Concepto de salud mental positiva: Factores relacionados. In J. Fornes, y J. Gómez (coord.), Recursos y programas para la salud mental. Enfermería psicosocial II, 37-69.

Loureiro, A.C. (2018). Literacia e saúde mental positiva: Tradução e validação de um instrumento de avaliação em literacia de saúde mental. (Dissertação de Mestrado). Escola Superior de Enfermagem do Porto. Acedido em https://comum.rcaap.pt/bitstream/10400.26/25783/1/Literacia\%20e\%20SMP.pdf 
Loureiro, L.M.J. (2015). Questionário de Avaliação da Literacia em Saúde Mental - QuALiSMental: estudo das propriedades psicométricas. Revista de Enfermagem Referência, Série IV - n. ${ }^{\circ}$ - jan./fev./mar. 2015, 79-88. Acedido em http://www.scielo.mec.pt/pdf/ref/vserIVn4/serIVn4a09.pdf

Murcho, N., Pacheco, E., \& Jesus, S.N. de (2016). Transtornos mentais comuns nos cuidados de saúde primários: um estudo de revisão. Revista Portuguesa de Enfermagem de Saúde Mental; 15, 30-34. http://dx.doi.org/10.19131/rpesm.0129

O'Connor, M., Sanson, A.V., Toumbourou, J.W., Norrish, J., \& Olsson, C.A. (2017). Does positive mental health in adolescence longitudinally predict healthy transitions in young adulthood? Journal of Happiness Studies, 18(1), 177-198 D0I: https://doi.org/10.1007/s10902-016-9723-3

Oliveira, S.V. (2013). A qualidade de sono dos enfermeiros. Universidade Fernando Pessoa. Faculdade de $\begin{array}{llll}\text { Ciências de } & \text { Saúde. } & \text { Acedido }\end{array}$ https://bdigital.ufp.pt/bitstream/10284/4056/1/Projecto\%20Gradua\%C3\%A7\%C3\%A30.pdf

Pedro, A. R.; Amaral, 0., \& Escoval, A. (2016). LS, dos dados à ação: Tradução, validação e aplicação do European health literacy survey em Portugal. Revista Portuguesa de Saúde Pública, 34(3), 259275.doi:http://dx.doi.org/10.1016/j.rpsp.2016.07.002.

Rosa, A., Loureiro, L., \& Sequeira, C. (2014). Literacia em saúde mental de adolescentes: Um estudo exploratório. Revista Portuguesa de Enfermagem de Saúde Mental (Ed. Esp. 1), 125-132.

Sequeira, C.J., Carvalho, C., Gonçalves, A., Nogueira, M.J, Lluch-Canut, T., \& Roldán-Merino, J. (2019). Levels of Positive Mental Health in Portuguese and Spanish Nursing Students. Journal of the American Psychiatric Nurses Association; 1-10. DOI: 10.1177/1078390319851569 journals.sagepub.com/home/jap 
